Pacific Journal of Mathematics

FURTHER RESULTS IN THE THEORY OF MONODIFFRIC
FUNCTIONS 


\title{
FURTHER RESULTS IN THE THEORY OF MONODIFFRIC FUNCTIONS
}

\author{
G. J. KURowskI
}

This paper considers two fundamental problems in the theory of monodiffric functions; i.e., discrete functions which satisfy the partial difference equation

$$
f(z+1)-f(z)=-i[f(z+i)-f(z)]
$$

on some region of the discrete $z$-plane, $z=m+i n, m=0$, $\pm 1, \pm 2, \cdots, n=0, \pm 1, \pm 2, \cdots$, and which, accordingly, are analogs of analytic functions.

The first problem considered centers about a process analogous to multiplication. A method of analytic extension is presented whereby a function defined along the real axis may be uniquely extended into the upper-half plane as a monodiffric function. The generalized product of two monodiffric functions may then be defined as the extension of a suitable product on the real axis. This definition is shown to be consistent with prior results.

The second problem is concerned with an analog to the Cauchy integral based upon a discrete singularity function which tends to zero as $|z|$ becomes large. The desired singularity function is obtained and the analogous integral formula presented.

Introduction and notation. In recent years, considerable attention has been given to the development of discrete and semi-discrete analogs of analytic functions (see, for example, references [1] through [4], [6]). The theory of these discrete or semi-discrete functions is obtained by deriving satisfactory analogs of classic results in the theory of analytic functions as well as finding results which have no direct analog in the classic theory.

The discrete analogs of analytic functions have been called by several names. Duffin [1] calls them discrete analytic functions, Ferrand [2] calls them preholomorphic functions, and Isaacs [3, 4] calls them monodiffric functions. In this paper we shall be using the definitions given by Isaacs which differ from those given by Duffin and Ferrand and, accordingly, we shall refer to these discrete analogs as monodiffric functions.

One central problem which has not been completely answered is the analog of a closed multiplication. That is, a procedure which is analogous to multiplication such that the "product" of two monodiffric 
functions would itself be a monodiffric function. Such analogs have been given, Isaacs [4], for the product of monodiffric functions with $z$. In this paper, we shall propose a method of analytic extension for monodiffric functions and apply this to define a generalized product of two monodiffric functions.

A second development in this paper concerns a monodiffric analog of the Cauchy integral formula. In [3], Isaacs obtains an analog of this formula based on a singularity function (called a wedge function) which tends to infinity with $|z|$. Accordingly, we will apply an operational calculus method, as used in [1] and [6], to obtain an integral formula based upon a singularity function which tends to zero as the modulus of $z$ tends to infinity.

Throughout this paper, we will be concerned with the monodiffric functions of the first kind as defined by Isaacs [3]. That is, we consider discrete functions $f(z)$ defined on the lattice of points $z=m+i n$ where $m=0, \pm 1, \pm 2, \cdots ; n=0, \pm 1, \pm 2, \cdots$ which satisfy the partial difference equation

$$
f(z+1)-f(z)=-i[f(z+i)-f(z)],
$$

for some range of values of $z$. It should again be noted that this definition is not the definition used by either Duffin [1] or Ferrand [2] in their treatises on discrete analytic functions.

In our discussion it will be convenient to use the translation operators

$$
\begin{array}{ll}
E_{1}^{k} f(z)=f(z+k) k>0 ; & E_{1}^{0} f(z)=f(z), \\
E_{2}^{k} f(z)=f(z+i k) k>0 ; & E_{2}^{0} f(z)=f(z),
\end{array}
$$

and the difference operators

$$
\begin{aligned}
& \Delta_{1} f(z)=f(z+1)-f(z), \\
& \Delta_{2} f(z)=f(z+i)-f(z) .
\end{aligned}
$$

Defining the operator $L$ by the relation

$$
L f(z)=\left[(1+i) I-E_{1}^{1}-i E_{2}^{1}\right] f(z),
$$

where $I f(z)=f(z)$, enables us to rewrite the defining equation (1.1) in the form

$$
L f(z)=0 .
$$

Additional notation will be introduced as required.

2. Analytic extension for monodiffric functions. Isaacs [3] defines a discrete function $f(z)$ on the lattice to be monodiffric of the 
first type at the point $z$ if it satisfies the defining equation (1.1) there. Since we shall consider only such functions, we shall refer to them simply as monodiffric functions.

This defining equation (1.1) is easily solved by Boole's method [5]. The solution is, in terms of an arbitrary function $g(x)$

$$
G(z)=\left[i E_{1}^{1}+I\right]^{y} g(x) ; \quad y \geqq 0 .
$$

We rewrite this solution by expanding the indicated operator using the binomial theorem. Thus,

$$
G(z)=\sum_{k=0}^{y} i^{k}\left(\begin{array}{c}
y \\
k
\end{array}\right) \Delta_{1}^{k} g(x),
$$

where $\left(\begin{array}{l}y \\ k\end{array}\right)$ denotes the binomial coefficient. However, since $\left(\begin{array}{l}y \\ k\end{array}\right)=0$ whenever $k>y$, an equivalent expression is

$$
G(z)=\sum_{k=0}^{\infty} i^{k}\left(\begin{array}{l}
y \\
k
\end{array}\right) \Delta_{1}^{k} g(x) ; \quad y \geqq 0 .
$$

This summation for a given integral value of $y$ is a finite sum because of the noted property of the binomial coefficients. Hence, convergence of (2.1) is assured for all finite $z$.

Equation (2.1) defines the analytic extension of $g(x)$ into the upper half plane of the lattice. That is, $G(z)$ is a monodiffric function whose values on the real axis are $g(x)$. By the defining equation (1.1), $G(z)$ is uniquely determined.

Let us consider an application of (2.1). We introduce the factorial polynomials

$$
\begin{aligned}
& (x)_{n}=x(x-1) \cdots(x-n+1), \\
& (y)_{n}=y(y-1) \cdots(y-n+1) .
\end{aligned}
$$

Using the well-known [5] result

$$
\Delta_{1}^{k}(x)_{n}=(n)_{k}(x)_{n-k},
$$

we obtain as the extension of $g(x)=(x)_{n}$

$$
G(z)=\sum_{k=0}^{n} i^{k}\left(\begin{array}{l}
n \\
k
\end{array}\right)(y)_{k}(x)_{n-k} .
$$

Equation (2.3) is recognized as the definition given by Isaacs [3] for the monodiffric pseudo-power $z^{(n)}$. Thus, we conclude that these pseudopowers of $z$ may be obtained by the analytic extension of the factorial polynomial $(x)_{n}$ into the lattice.

Analytic extension can be thought of as the application of the operator 


$$
\mathscr{E}=\sum_{k=0}^{\infty} i^{k}\left(\begin{array}{l}
y \\
k
\end{array}\right) \Delta_{1}^{k}
$$

(we may call $\mathscr{E}$ the extension operator) to a function $g(x)$ on the real line; i.e., we could write (2.1) in the form $G(z)=\mathscr{E}[g(x)]$. Clearly $\mathscr{E}$ is a linear operator and consequently linear functional identities are preserved by $\mathscr{E}$. For example, if $S^{(2)}(z)=\mathscr{E}\left[\sin ^{2} x\right]$ and $C^{(2)}(z)=$ $\mathscr{E}\left[\cos ^{2} x\right]$, these extensions satisfy the identity

$$
S^{(2)}(z)+C^{(2)}(z)=1 .
$$

3. Multiplication of monodiffric functions. The method of analytic extension (2.1) enables us to specify for monodiffric functions a process analogous to multiplication. Accordingly, we define the generalized product, denoted by $F(z) \cdot G(z)$, of two monodiffric functions $F(z)$ and $G(z)$, which are the extensions of $f(x)$ and $g(x)$ respectively, to be the extension of the product $f(x) g(x-1)$. That is,

$$
F(z) \cdot G(z)=\sum_{k=0}^{\infty} i^{k}\left(\begin{array}{l}
y \\
k
\end{array}\right) \Delta_{1}^{k}[f(x) g(x-1)]
$$

Equation (3.1) may be transformed into a more convenient form on using the finite-difference analog of Leibnitz's rule

$$
\Delta_{1}^{k}[f(x) g(x-1)]=\sum_{m=0}^{k}\left(\begin{array}{c}
k \\
m
\end{array}\right) \Delta_{1}^{m} f(x) \Delta_{1}^{k-m} g(x-1+m) .
$$

Algebraic manipulation of (3.1) using (3.2) and the relation

$$
(y)_{m}=(y)_{p}(y-p)_{m-p}
$$

enables us to write (3.1) in the form

$$
F(z) \cdot G(z)=\sum_{p=0}^{\infty} i^{p}\left(\begin{array}{l}
y \\
p
\end{array}\right) \Delta_{1}^{p} f(x) \sum_{n=0}^{\infty} i^{n}(y-p) \Delta_{1}^{n} g(x+p-1) .
$$

However, since $G(z)$ is the extension of $g(x)$, we have

$$
G(z-1+p(1-i))=\sum_{n=0}^{\infty} i^{n}(y-p) \Delta_{1}^{n} g(x+p-1) .
$$

Substitution of (b) into (a) gives the final result,

$$
F(z) \cdot G(z)=\sum_{p=0}^{\infty} i^{p}\left(\begin{array}{l}
y \\
p
\end{array}\right) G(z-1+p[1-i]) \Delta_{1}^{p} f(x) .
$$

Equation (3.3) will be taken as the definition for the generalized product $F(z) \cdot G(z)$. By construction, this product is monodiffric.

In [4], Isaacs defines a multiplication $z \cdot G(z)$ of a monodiffric function $G(z)$ by $z$ with the following relation: 


$$
z \cdot G(z)=x G(z-1)+i y G(z-i) .
$$

If, in (3.3), we take $f(x)=x$, whose extension is $z=z^{(1)}$, we obtain (3.4). Thus, the definition of this generalized product (3.3) is consistent with the definition (3.4) given by Isaacs for multiplication by $z$. In particular, this implies that the iterative definition

$$
z \cdot z^{(n)}=z^{(n+1)}, \quad n \geqq 0 ; \quad z^{(0)}=1,
$$

for the generation of the pseudo-powers of $z$ is consistent with the definition (3.3).

In order to extend Isaacs' definition (3.4) for the product $z \cdot G(z)$, we have defined $F(z) \cdot G(z)=\mathscr{E}[f(x) g(x-1)]$. However, since $G(z) \cdot F(z)=$ $\mathscr{E}[g(x) f(x-1)]$, this generalized product is not commutative. A similar analysis shows that this generalized product is not associative. This product is distributive over addition

$$
\begin{aligned}
& F(z) \cdot[G(z)+H(z)]=F(z) \cdot G(z)+F(z) \cdot H(z), \text { and } \\
& {[G(z)+H(z)] \cdot F(z)=G(z) \cdot F(z)+H(z) \cdot F(z) .}
\end{aligned}
$$

A second generalized product defined by $F(z) \otimes G(z)=\mathscr{E}[f(x) g(x)]$ is both commutative and associative. However, this definition is not consistent with Isaacs' result (3.4). It is easily established that

$$
z \otimes G(z)=\mathscr{E}[x g(x)]=x G(z)+i y G(z+1-i) .
$$

4. Monodiffric analog of the Cauchy integral formula. In [3], Isaacs obtains an analog of the Cauchy integral formula based upon a singularity function which tends to infinity with $|z|$. Accordingly, in this section we shall reconsider this question of an analog for the integral formula and develop one based upon a singularity function which tends to zero as $|z|$ becomes large.

Before we can proceed with this development, it is advisable to recall some of the definitions given by Isaacs and to introduce some new terminology. Isaacs [3] defines the conjoint path sum along the discrete path $P$, denoted by

$$
\sum_{(P)} f(z), g(z) \Delta z
$$

as follows:

(a) The contribution to (4.1) in going from $z$ to $z+1$ is $f(z) g(z+1)$,

(b) The contribution to (4.1) in going from $z$ to $z+i$ is if $(z) g(z+i)$,

(c) The contribution to (4.1) in going from $b$ to $a$ is the negative 
of the contribution to (4.1) in going from $a$ to $b$. Corresponding to the operator $L$ which defines monodiffric functions, we introduce the operator $B$ by the relation

$$
B=(1+i) I-E_{1}^{-1}-i E_{2}^{-1} .
$$

A discrete function $g(z)$ is said to be co-monodiffric at the point $z$ if $B g(z)=0$. It is co-monodiffric on a domain $D$ of the lattice if it is co-monodiffric at every point of $D$.

A basic square of the lattice, identified by the point $\alpha$ in its lower left corner and denoted by $S(\alpha)$ is the set

$$
S(\alpha)=\{\alpha+(k+i n) ; k=0,1 ; n=0,1\} .
$$

The exterior boundary $P(S)$ of $S(\alpha)$ is the set

$$
P(S)=\left\{\begin{array}{l}
\alpha+i k, \quad \alpha+2+i k ; \quad k=0,1,2 \\
\alpha+1+i m ; \quad m=0,2
\end{array}\right\} .
$$

The point $\{\alpha+i+1\}$ of the basic square $S(\alpha)$ is said to be the interior of $S(\alpha)$, denoted by $I(\alpha)$.

A domain $D$ of the lattice is a finite union of basic squares $S\left(\alpha_{j}\right)$. The interior $I$ of $D$ is the union of the interior points of the basic squares $S\left(\alpha_{j}\right)$ comprising $D$. The exterior boundary of $D$, denoted by $P$, is the union of the exterior boundaries of the composite basic squares of $D$ intersected with the complement $\widetilde{I}$ of $I$. That is,

$$
D=\bigcup_{\jmath} S\left(\alpha_{j}\right)
$$

and

$$
I=\bigcup_{j} I\left(\alpha_{j}\right) ; \quad P=\left[\bigcup_{j} P\left(\alpha_{j}\right)\right] \cap \widetilde{I} .
$$

The following diagram illustrates these definitions for a domain $D$ composed of two basic squares (shaded). To facilitate the example, we let $k$ stand for the point $z_{k}$.

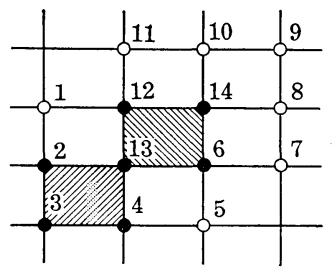

$$
\begin{aligned}
D & =\{2,3,4,136,12,13,14\} \\
I & =\{13,14\} \\
P & =\{1,2, \cdots, 11,12\} .
\end{aligned}
$$

A closed path $P$ is said to include a basic square $S(\alpha)$ if all the vertices of $S(\alpha)$ are points of the domain determined by $P$. With these definitions, the following theorems are readily established for a basic square $S(\alpha)$. Their extension to domains $D$ given by $(4.5)$ follows by juxtaposition. 
THEOREM 1. [Green's Identity] Let $f(z), g(z)$ be lattice functions defined on a domain $D$ and its exterior boundary $P$. Then,

$$
\begin{gathered}
i \sum_{D} \sum\{f(z) B g(z+1+i)+g(z+1+i) L f(z)\} \\
=\sum_{(P)} f(z), g(z) \Delta z .
\end{gathered}
$$

THEOREM 2. [Cauchy Integral Theorem] If $f(z)$ is monodiffric and $g(z)$ is co-monodiffric on the domain $D$ and if $P$ is any closed path contained in $D$, then

$$
\sum_{(P)} f(z), g(z) \Delta z=0 .
$$

The formula (4.8) was obtained by Isaacs [3] in a somewhat different context. He defined those pairs of functions $f, g$ which satisfied (4.8) on a basic square to be conjoint monodiffric and proved that if $f(z)$ and $g(z)$ are conjoint monodiffric, then (4.8) is valid over a closed path in $D$ and further that if (4.8) is valid over all closed paths, then $f(z)$ and $g(z)$ are conjoint monodiffric on the domain. By virtue of Theorem 1 and 2 above, we have the following:

THEOREM 3. The discrete functions $f(z)$ and $g(z)$ are conjoint monodiffric on a domain $D$ if $f(z)$ is monodiffric and $g(z)$ is comonodiffric on $D$.

To develop an analog of the Cauchy integral formula, the Green's identity (4.7) enables us to specify desirable properties for a singularity function. Thus, we seek a function $G(z)$ such that

(i) $B G(z)=0$ for all $z$ of the lattice except the point $z=1+i$ where $B G(1+i)=1$.

(ii) $|G(z)|$ tends to zero as $|z|$ becomes large.

For the moment, let us ignore the second of these properties for $G(z)$ and solve the difference equation

$$
B h(z)=\left\{\begin{array}{lll}
0 & \text { if } & x \neq 0, \quad y \neq 0 \\
1 & \text { if } \quad x=y=0
\end{array}\right.
$$

The solution to (4.9) could readily be translated to satisfy property (i). Equations (4.9) is solved using the method of generating functions. Thus we define

$$
\text { ( a) } H(u, t)=\sum_{x} \sum_{y} h(z) u^{x} t^{y} .
$$

Substitution into $(4.9)$ results in

$$
\text { (b) } H(u, t)=[(1-u)+i(1-t)]^{-1} \text {. }
$$


Expanding (b) in a power series and comparing with (a) gives the following

$$
h(z)=\left\{\begin{array}{c}
\left(\begin{array}{c}
x+y \\
y
\end{array}\right) i^{y}(1+i)^{-(x+y+1)} ; x \geqq 0, \quad y \geqq 0 \\
0, \text { all other values of } x, y .
\end{array}\right.
$$

Investigation of $h(z)$ shows that property (ii) is violated and further shows that $h(z)$ is a wedge function as defined by Issacs [3]. Accordingly, $h(z)$ together with Theorem 1 results in one of the analogs of the Cauchy integral formula stated by Isaacs. However, $h(z)$ does not possess the desired property (ii) for large values of $|z|$.

In order to obtain a singularity function having both properties (i) and (ii), we adopt the methods of the operational calculus as used by Duffin [1], Kurowski [6] and others. Such considerations lead immediately to the following function (whose singular point is $z=0$ )

$$
G(z ; 0)=(2 \pi)^{-2} \int_{-\pi}^{\pi} \int_{-\pi}^{\pi} e^{i(a x+b y)} Q(a, b) d a d b,
$$

where

$$
Q(a, b)=\left[\left(1-e^{-i a}\right)+i\left(1-e^{-i b}\right)\right]^{-1} .
$$

It is easily verified that $G(z ; 0)$ satisfies requirement (i) for the singular point $z=0$. Since $G(z ; 0)$ can be regarded as being the Fourier coefficients of $Q(a, b)$, an integrable function on the indicated square of integration, $G(z ; 0)$ tends to vanish as $|z|$ becomes large by the Riemann-Lebesgue theorem. To obtain the singularity function $G(z ; 1+i)$ which satisfies property (i), we replace $x$ by $x-1$ and $y$ by $y-1$ in (4.11). By the remarks above, $G(z ; 1+i)$ has both of the desired properties (i) and (ii). By (4.7) we have the following analog of the Cauchy integral formula.

THEOREM 4. Let $f(z)$ be monodiffric on a domain $D$ of the lattice which includes the basic square $S(0)$. Let $G(z ; 1+i)$ be the singularity function defined in (4.11) with $x$ replaced by $(x-1)$ and $y$ replaced by $(y-1)$. If $P$ is any closed path in $D$ which includes $S(0)$, then

$$
\sum_{(P)} f(z), G(z ; 1+i) \Delta z=i f(0),
$$

Otherwise the value of this conjoint path sum is zero.

An elementary translation of the parametric point $(1+i)$ of the singularity function $G(z ; \lambda)$ yields the general analog of the Cauchy integral formula. 


\section{REFERENCES}

1. R. J. Duffin, Basic properties of discrete analytic functions, Duke Math. J. 23 (1956), 335-364.

2. J. Ferrand, Fonctions préharmoniques et fonctions préholomorphes, Bull. Sci. Math. 68 (1944), second series, 152-180.

3. R. P. Isaacs, A finite difference function theory, Univ. Nac. Tucumán Rev., 2 (1941), 177-201.

4. - Monodiffric functions, Nat. Bur. Standards Appl. Math. Ser. 18 (1952), $257-266$.

5. C. Jordan, Calculus of Finite Differences, 2nd ed., Chelsea, New York, 1950.

6. G. J. Kurowski, Semi-discrete analytic functions, Trans. Amer. Math. Soc. 106 (1963), 1-18.

Received December 9, 1964.

UNIVERSITY OF CALIFORNIA, DAVIS 



\section{PACIFIC JOURNAL OF MATHEMATICS}

H. SAMELSON

Stanford University Stanford, California

R. M. BLUMENTHAL

University of Washington

Seattle, Washington 98105

\section{EDITORS}

\author{
*J. DugundJI \\ University of Southern California \\ Los Angeles, California 90007 \\ RICHARD ARENS \\ University of California \\ Los Angeles, California 90024
}

\section{ASSOCIATE EDITORS}

E. F. BeCKENBACH

B. H. NEUMANN

F. WOLF

K. YosidA

\section{SUPPORTING INSTITUTIONS}

UNIVERSITY OF BRITISH COLUMBIA

CALIFORNIA INSTITUTE OF TECHNOLOGY

UNIVERSITY OF CALIFORNIA

MONTANA STATE UNIVERSITY

UNIVERSITY OF NEVADA

NEW MEXICO STATE UNIVERSITY

OREGON STATE UNIVERSITY

UNIVERSITY OF OREGON

OSAKA UNIVERSITY

UNIVERSITY OF SOUTHERN CALIFORNIA
STANFORD UNIVERSITY

UNIVERSITY OF TOKYO

UNIVERSITY OF UTAH

WASHINGTON STATE UNIVERSITY

UNIVERSITY OF WASHINGTON

AMERICAN MATHEMATICAL SOCIETY CHEVRON RESEARCH CORPORATION TRW SYSTEMS

NAVAL ORDNANCE TEST STATION 


\section{Pacific Journal of Mathematics}

Vol. 18, No. 1

March, 1966

Edward Joseph Barbeau, Semi-algebras that are lower semi-lattices ...... 1

Steven Fredrick Bauman, The Klein group as an automorphism group

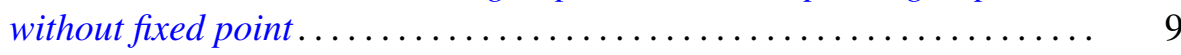

Homer Franklin Bechtell, Jr., Frattini subgroups and $\Phi$-central groups .... 15

Edward Kenneth Blum, A convergent gradient procedure in prehilbert

spaces ............................................

Edward Martin Bolger, The sum of two independent exponential-type random variables ...................................

David Wilson Bressler and A. P. Morse, Images of measurable sets .......

Dennison Robert Brown and J. G. LaTorre, A characterization of uniquely

divisible commutative semigroups........................

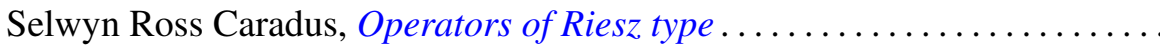

Jeffrey Davis and Isidore Isaac Hirschman, Jr., Toeplitz forms and ultraspherical polynomials ............................

Lorraine L. Foster, On the characteristic roots of the product of certain rational integral matrices of order two ......................

Alfred Gray and S. M. Shah, Asymptotic values of a holomorphic function

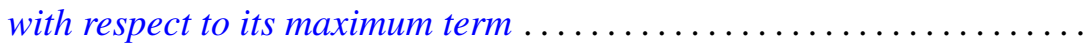

Sidney (Denny) L. Gulick, Commutativity and ideals in the biduals of

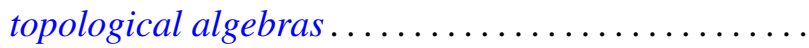

G. J. Kurowski, Further results in the theory of monodiffric functions

Lawrence S. Levy, Commutative rings whose homomorphic images are self-injective .

Calvin T. Long, On real numbers having normality of order $k$....

Bertram Mond, An inequality for operators in a Hilbert space. ...

John William Neuberger, The lack of self-adjointness in three-point boundary value problems ........................

C. A. Persinger, Subsets of $n$-books in $E^{3}$

Oscar S. Rothaus and John Griggs Thompson, A combinatorial problem in the symmetric group ............................... 175

Rodolfo DeSapio, Unknotting spheres via Smale .................. 179

James E. Shockley, On the functional equation

$$
F(m n) F((m, n))=F(m) F(n) f((m, n)) \ldots \ldots \ldots
$$

Kenneth Edward Whipple, Cauchy sequences in Moore spaces ... 